\title{
Neural Network Interactions Modulate CRY-Dependent Photoresponses in Drosophila
}

\author{
Pallavi Lamba, Lauren E. Foley, and $\odot$ Patrick Emery \\ Department of Neurobiology, and Program in Neuroscience, Graduate School of Biomedical Sciences, University of Massachusetts Medical School, 364 \\ Plantation Street, Worcester, Massachusetts 01605
}

Light is one of the chief environmental cues that reset circadian clocks. In Drosophila, CRYPTOCHROME (CRY) mediates acute photic resetting of circadian clocks by promoting the degradation of TIMELESS in a cell-autonomous manner. Thus, even circadian oscillators in peripheral organs can independently perceive light in Drosophila. However, there is substantial evidence for nonautonomous mechanisms of circadian photoreception in the brain. We have previously shown that the morning $(\mathrm{M})$ and evening $(\mathrm{E})$ oscillators are critical light-sensing neurons that cooperate to shift the phase of circadian behavior in response to light input. We show here that light can efficiently phase delay or phase advance circadian locomotor behavior in male Drosophila even when either the M- or the E-oscillators are ablated, suggesting that behavioral phase shifts and their directionality are largely a consequence of the cell-autonomous nature of CRY-dependent photoreception. Our observation that the phase response curves of brain and peripheral oscillators are remarkably similar further supports this idea. Nevertheless, the neural network modulates circadian photoresponses. We show that the M-oscillator neurotransmitter pigment dispersing factor plays a critical role in the coordination between $\mathrm{M}$ - and E-oscillators after light exposure, and we uncover a potential role for a subset of dorsal neurons in the control of phase advances. Thus, neural modulation of autonomous light detection might play an important role in the plasticity of circadian behavior.

Key words: behavior; circadian; Drosophila; photoreception

Significance Statement

Input pathways provide circadian rhythms with the flexibility needed to harmonize their phase with environmental cycles. Light is the chief environmental cue that synchronizes circadian clocks. In Drosophila, the photoreceptor CRYPTOCHROME resets circadian clocks cell-autonomously. However, recent studies indicate that, in the brain, interactions between clock neurons are critical to reset circadian locomotor behavior. We present evidence supporting the idea that the ability of flies to advance or delay their rhythmic behavior in response to light input essentially results from cell-autonomous photoreception. However, because of their networked organization, we find that circadian neurons have to cooperate to reset the phase of circadian behavior in response to photic cues. Our work thus helps to reconcile cell-autonomous and non-cell-autonomous models of circadian entrainment.

\section{Introduction}

Circadian clocks are endogenous time-keeping mechanisms that drive rhythms in behavior, physiology, and gene expression. The

Received Aug. 9, 2017; revised April 17, 2018; accepted May 2, 2018.

Author contributions: P.L. and P.E. designed research; P.L. and L.E.F. performed research; P.L. and P.E. analyzed data; P.L. and P.E. wrote the paper.

This work was supported by National Institutes of Health Maximizing Investigators' Research Award (R35) \#GM118087 from the National Institute of General Medical Sciences. We thank Diana Wentworth, Elaine Chang, and Diane Szydlik for technical support; and the Emery and Weaver laboratory members for helpful discussions. We also thank David Weaver and Yong Zhang for critically reading our manuscript. In addition, we thank Dr. Paul Taghert for Pdf ${ }^{\circ}$ flies; Dr. Michael Rosbash for DvPdf-GAL4; Pdf-GAL80 and tubGAL80'5; UAS-kir; and the TRiP stock center for jet RNAi transgenic flies.

The authors declare no competing financial interests.

Correspondence should be addressed to Dr. Patrick Emery, Department of Neurobiology, and Program in Neuroscience, Graduate School of Biomedical Sciences, University of Massachusetts Medical School, 364 Plantation Street, LRB 703, Worcester, MA 01605. E-mail: Patrick.Emery@umassmed.edu. ability of circadian clocks to be reset by various environmental cues allows organisms to anticipate changes in the environment and capitalize on available resources. Light is the cardinal synchronizer of circadian clocks; it entrains them to the $24 \mathrm{~h}$ solar cycle. In a $12 \mathrm{~h} / 12 \mathrm{~h}$ light/dark (LD) cycle, Drosophila exhibits a bimodal activity pattern. A morning peak of activity in anticipation of lights-on is generated by small ventrolateral neurons (sLNvs), also referred to as the morning (M) oscillators (Grima et al., 2004; Stoleru et al., 2004). The sLNvs are also the pacemaker neurons that maintain free-running behavioral rhythms in constant conditions by rhythmically secreting pigment dispersing factor (PDF) to synchronize the other circadian neurons (Renn et al., 1999; Stoleru et al., 2005). The evening peak of activity in anticipation of 
lights-off is governed by the dorsolateral neurons (LNds) and the PDF-negative fifth sLNvs, together referred to as the evening (E) oscillators (Grima et al., 2004; Stoleru et al., 2004).

Drosophila circadian clocks can perceive light via conventional photoreceptive organs: the compound eyes, the ocelli, the Hofbauer-Buchner eyelet, and the cell-autonomous photoreceptors CRYPTOCHROME (CRY) and Rhodopsin-7 (Emery et al., 1998; Stanewsky et al., 1998; Helfrich-Förster et al., 2001; Rieger et al., 2003; Ni et al., 2017). Mechanistically, the CRY-dependent input pathway is best understood. CRY resets the pacemaker by promoting the rapid degradation of the key pacemaker protein TIMELESS (TIM; Lin et al., 2001). Upon light exposure, CRY undergoes a conformational change that enables its binding to TIM and promotes TIM degradation via the F-box protein JETLAG (JET), a component of an E3 ubiqitin ligase (Busza et al., 2004; Koh et al., 2006). The Drosophila circadian clock is very sensitive to light. A brief light pulse in the early night, mimicking a belated dusk, delays the phase of circadian behavior, while a late-night pulse is perceived as an early dawn and thus advances the phase of locomotor activity (Levine et al., 1994). Severe cry or jet mutants are unable to respond to short light pulses, and acute TIM degradation is impaired (Stanewsky et al., 1998; Lin et al., 2001; Lamba et al., 2014). The molecular mechanism underlying CRY-dependent photoresponses is thus cell autonomous. In fact, it has been shown that circadian oscillators in isolated body segments such as wings, legs, and antennae can sense light independently and re-entrain to a new LD cycle shifted by $6 \mathrm{~h}$ (Plautz et al., 1997). However, in the brain, there is strong evidence for additional nonautonomous mechanisms. First, CRY expression limited to the $\mathrm{M}$-oscillators is not sufficient to fully rescue circadian phase shifts in cry mutants (Emery et al., 2000; Lamba et al., 2014). Second, not all circadian neurons express CRY, yet even CRY-negative circadian neurons can acutely degrade TIM in a CRY-dependent manner (Yoshii et al., 2008). Third, specific groups of circadian neurons have been proposed to promote phase delays (DN1s) or phase advances (lLNvs); Shang et al., 2008; Tang et al., 2010). Fourth, we have recently shown that the $\mathrm{M}$ - and E-oscillators are the critical light-sensing neurons, working together to reset the phase of circadian behavior upon light exposure (Lamba et al., 2014). Moreover, we found that JET in M-oscillators could promote non-cell-autonomously acute TIM degradation in E-oscillators. Since neural cooperation between $\mathrm{M}$ - and E-oscillators is required for photic resetting, we surmised that these circadian neurons are exchanging signals during or after light exposure to cause behavioral phase shifts.

Unexpectedly, we show here that neither the M-oscillators nor the E-oscillators are required for behavioral phase delays or advances in responses to light pulses. However, when both cell groups are present, the M-oscillator neurotransmitter PDF functions as a modulator of CRY-dependent light detection. Our results also suggest that a subset of dorsal neurons (DNs) are able to modulate specifically phase advances.

\section{Materials and Methods}

Fly stocks. Flies were raised on cornmeal/agar medium at $25^{\circ} \mathrm{C}$ under an LD cycle. For the experiments in which E-oscillators are conditionally silenced only during adulthood using tubGAL80 ${ }^{t s}$, the GAL4 and UAS controls as well as the experimental flies were raised at $18^{\circ} \mathrm{C}$ (permissive temperature for $G A L 80^{t s}$ ) until eclosion, after which the flies were transferred to $29^{\circ} \mathrm{C}$. The following Drosophila strains were used in this study: $y w ; w^{1118} ; y w ;$ Pdf-GAL4 (Renn et al., 1999; RRID:BDSC_6900); $y w$; Pdf-GAL4 UAS-dicer2 (Dubruille et al., 2009); y w; Pdf-GAL80; cryGAL4(13); y w; UAS-hid ${ }^{14} ; y$ w; UAS-hid; Pdf-GAL80 (Stoleru et al., 2004); $y$ w; cry-GAL4-13 (Emery et al., 2000); and jet RNAi (TRiP.
JF01506, Bloomington Drosophila Stock Center, Indiana University Bloomington, Bloomington, IN). The y w; $P d f^{0}$ (RRID:BDSC_26654; Renn et al., 1999) and pdfr mutant $\left(\right.$ han $\left.^{5304}\right)$ flies (RRID:BDSC_33068; Hyun et al., 2005) were a gift from Dr. Paul Taghert (Washington University in St. Louis, St. Louis, MO). The DvPdf-GAL4; Pdf-GAL80 and tubGAL80 ${ }^{\text {ts }}$; UAS-kir lines (Guo et al., 2014) were a gift from Dr. Michael Rosbash (Brandeis University, Waltham, MA). The jet RNAi line was genetically recombined with $P d f^{0}$, and $y w ; P d f$-GAL4 UAS-dicer 2 was combined with the $p d f r$ mutation. The presence of $s$-tim or $l s$-tim alleles (Rosato et al., 1997) was determined by PCR and sequencing. Within each experiment, the experimental and control flies carried the same tim alleles, with the exception of the conditional silencing experiments in which the experimental and the GAL4 controls were $l s$-tim homozygous, while the UAS control flies were $l s$-tim/s-tim heterozygotes. Transgenic flies expressing a tim-luciferase fusion gene under the control of the tim promoter $(\sim 5 \mathrm{~kb})$ and first intron (ptim-TIM-LUC) were used for luciferase (LUC) experiments.

Behavioral monitoring and analysis. Single adult male (2- to 5-d-old) flies were used to test locomotor activity. Groups of 16 flies per genotype, or 32 flies for genotypes with high arrhythmicity, were entrained to a 12 $\mathrm{h} / 12 \mathrm{~h} \mathrm{LD}$ cycle for $4-5 \mathrm{~d}$ at $25^{\circ} \mathrm{C}$, except for the conditional silencing

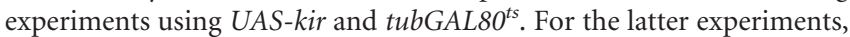
flies were entrained to an $\mathrm{LD}$ cycle for $6-7 \mathrm{~d}$ at $20^{\circ} \mathrm{C}$ or $29^{\circ} \mathrm{C}$ to repress or induce the inward-rectifier potassium channel KIR expression, respectively. After entrainment, flies were exposed to a 5 min pulse of white fluorescent light (1500 lux) at different time points on the last night of the LD cycle. A separate control group of flies was not light pulsed. Following the light pulse, flies were released in constant darkness (DD) and their locomotor activity was monitored using the TriKinetics activity monitors for $6 \mathrm{~d}$. Rhythmicity and period length were analyzed using the FaasX software (gift from F. Rouyer, Centre National de la Recherche Scientifique, Gif-sur-Yvette, France). Rhythmicity was defined by the following criteria: power $\geq 10$, width $\geq 2$ using the $\chi^{2}$ periodogram analysis (Ewer et al., 1992). To determine the amplitude of photic phase shifts, data analysis was performed in Excel (Microsoft) using activity data from all flies, including those that were arrhythmic, according to periodogram analysis. Activity was averaged within each group of 16 or 32 flies, plotted in Excel, and then fitted with a $4 \mathrm{~h}$ moving average. A genotype-blind observer quantified the phase shifts. The peak of activity was found to be the most reliable phase marker for all genotypes except for flies mutant for $p d f r$. For these flies, the activity trough was obviously a much more reliable phase marker. Phase shifts were calculated by subtracting the average peak (or trough) phase of the light-pulsed group from the average peak (or trough) phase of a non-light-pulsed group of flies. For genotypes with high arrhythmicity in DD, the phase was measured only from the days during which the flies showed clear rhythmic activity as a population.

Luciferase experiments. The luciferase activity of ptim-TIM-LUC flies on luciferin (Gold Biotechnology) containing agar/sucrose medium (170 $\mu \mathrm{l}$ volume, $1 \%$ agar, $2 \%$ sucrose, $25 \mathrm{~mm}$ luciferin), was monitored in a Centro LB 960 Microplate Luminometer (Berthold Technologies) in I-36LL Incubators (Percival Scientific) with 90\% humidity. Flies in 96well white plates were covered with needle-poked Pattern Adhesive PTFE Sealing Film (catalog \#961801, Analytical Sales \& Services). The distance between the agar and the film was such that the flies were not able to move vertically. Light pulse protocol and phase analysis was the same as for locomotor activity. Raw bioluminescence data were treated as described for behavioral data, and phase shifts were determined by comparing the phase of the first peak of bioluminescence in DD in pulsed and nonpulsed flies. Amplitude in pulsed flies $\left(A_{\mathrm{p}}\right)$ and amplitude in nonpulsed flies $\left(A_{\mathrm{np}}\right)$ was calculated by dividing the bioluminescence peak value with the average of the value of the first two troughs in DD. The relative amplitude (RA) shown on the $y$-axis of Figure $6 C$ was calculated as follows: $\mathrm{RA}=\left(A_{\mathrm{p}}-1\right) /\left(A_{\mathrm{np}}-1\right)$. The subtraction from $A_{\mathrm{p}}$ and $A_{\mathrm{np}}$ accounts for the fact that an arrhythmic trace would have an amplitude equal to 1 .

To monitor luciferase activity in decapitated bodies, ptim-TIM-LUC flies were entrained to a $12 \mathrm{~h} / 12 \mathrm{~h}$ LD cycle for $3 \mathrm{~d}$, and bodies were severed from heads on the last day of the LD cycle in M3 medium (Shields 
Table 1. Circadian locomotor behavior under constant darkness

\begin{tabular}{|c|c|c|c|c|}
\hline Genotype & Flies $(n)$ & Rhythmic flies (\%) & Period average ( \pm SEM) & Power average $^{a}$ (士SEM) \\
\hline$p d f^{0}$ & 448 & 16 & $22.6 \pm 0.15$ & $36.52 \pm 2.33$ \\
\hline Pdf-GAL4/UAS-hid & 61 & 5 & $23 \pm 0.05$ & $36.1 \pm 10.95$ \\
\hline Pdf-GAL4/+ & 33 & 93 & $24.6 \pm 0.06$ & $57.03 \pm 2.54$ \\
\hline PD2/+ & 89 & 87 & $24.4 \pm 0.07$ & $50.06 \pm 3.43$ \\
\hline UAS-jet RNAi & 91 & 85 & $24 \pm 0.09$ & $62.2 \pm 5.21$ \\
\hline PD2/UAS-jet RNAi & 120 & 88 & $24.2 \pm 0.08$ & $49.9 \pm 2.34$ \\
\hline PD2, pdf ; UAS-jet RNAi, pdf & 240 & 7 & $22.9 \pm 0.15$ & $28.05 \pm 1.26$ \\
\hline UAS-hid;Pdf-GAL80/+ & 54 & 92 & $23.9 \pm 0.05$ & $83.8 \pm 14.6$ \\
\hline cry-GAL4(13)/+ & 40 & 77 & $23.8 \pm 0.03$ & $58.6 \pm 14.9$ \\
\hline UAS-hid;Pdf-GAL80/cry-GAL4(13) & 108 & 38 & $23.9 \pm 0.12$ & $48.05 \pm 7.95$ \\
\hline tub-GAL80's; UAS-kir/+ & 123 & 77 & $26.1 \pm 0.05$ & $49.9 \pm 3.95$ \\
\hline DvPdf-GAL4; Pdf-GAL80 & 121 & 94 & $24.9 \pm 0.09$ & $76.85 \pm 5.96$ \\
\hline tub-GAL80 ${ }^{t s} ; U A S-k i r / D v P d f-G A L 4 ; P d f-G A L 80$ & 220 & 32 & $25.8 \pm 0.13$ & $36.27 \pm 3.96$ \\
\hline Pdf-GAL80; cry-GAL4(13) & 38 & 100 & $23.5 \pm 0.05$ & $95.45 \pm 6.93$ \\
\hline tub-GAL80 ${ }^{\text {ts }}$, UAS-kir/Pdf-GAL80; cry-GAL4(13) & 73 & 16 & $26.7 \pm 0.11$ & $29.3 \pm 6.1$ \\
\hline Pdfr ${ }^{-} ;$PD2 & 95 & 47 & $23 \pm 0.03$ & $48.2 \pm 5.8$ \\
\hline Pdfr ${ }^{-}$; PD2; UAS-jet RNAi & 159 & 31 & $23.3 \pm 0.17$ & $35.6 \pm 3.6$ \\
\hline
\end{tabular}

${ }^{a}$ Power is a measure of rhythm amplitude and corresponds to the height of the periodogram peak above the significance line (Ewer et al., 1992). It was calculated from rhythmic flies only.

and Sang M3 insect medium, Sigma-Aldrich) $+12 \%$ fetal bovine serum $+1 \%$ penicillin-streptomycin $+0.1 \%$ insulin-transferrin-selenium +2 $\mu \mathrm{g} / \mathrm{ml}$ amphotericin-B $+25 \mathrm{~mm}$ luciferin). Twelve bodies were cultured together by placing them on a sterile Millicell Culture Plate Insert (Millipore) within a well of a Hamamatsu LM-2400 Luminometer. Cultures were monitored in the same solution throughout the experiment. Bioluminescence was recorded by the luminometer for $1 \mathrm{~min}$ at $15 \mathrm{~min}$ intervals. On the last night of the LD cycle, the light pulse was administered at zeitgeber time 15 (ZT15) for phase delay responses or at ZT23 for phase advance responses. Within each experiment, seven cultures were monitored for each time point and for the nonpulsed control in DD for $3 \mathrm{~d}$. The light pulse protocol and phase shift analysis were performed as described above for whole flies.

Statistical analysis. For the statistical analysis of the phase-shifting behavioral experiments, a Student's $t$ test was used to compare means between two groups, and a one-way ANOVA coupled to Tukey's post hoc test was used for multiple comparisons.

\section{Results}

\section{Light can reset the phase of circadian behavior in the absence of M-oscillators}

CRY photoreception functions independently in the brain and peripheral tissues. Peripheral tissues use CRY to entrain to the LD cycle, but they cannot entrain brain clock neurons if those are defective for CRY photoreception. Furthermore, rescuing CRY expression only in the circadian pacemaker neurons, the $\mathrm{M}$ cells, partially rescues behavioral photoresponses, but does not entrain peripheral clocks (Emery et al., 2000; Levine et al., 2002; Tang et al., 2010; Lamba et al., 2014). This reflects the cell-autonomous nature of CRY photoreception. However, in the brain, strong evidence suggest that nonautonomous mechanisms are also at play (Shang et al., 2008; Yoshii et al., 2008; Tang et al., 2010; Lamba et al., 2014). We therefore wanted to determine how the M-oscillators communicate with the E-oscillators during CRYdependent photoresponses. PDF is the principal circadian neurotransmitter responsible for synchronizing different groups of neurons and for the persistence of rhythmicity under freerunning conditions (Renn et al., 1999; Lin et al., 2004). Moreover, Guo et al. (2014) have shown that the induction of firing in PDFpositive neurons generates behavioral phase shifts reminiscent of those induced by light pulses and triggers TIM degradation in E-oscillators in a PDF receptor (PDFR)-dependent manner (Guo et al., 2014). Hence, PDF would be an ideal candidate for the signal transmitted from $\mathrm{M}$ - to E-oscillators during light-dependent phase resetting. $P d f^{0}$ flies show an advanced evening peak of activity in LD conditions and gradually become arrhythmic in DD (Renn et al., 1999). A small fraction of flies (16\% in our hands) remain rhythmic in DD with a short period of $\sim 22 \mathrm{~h}$ (Table 1 ). Hence, it was possible to test the ability of these flies to shift their behavior in response to delaying and advancing light pulses of saturating light intensity (Suri et al., 1998; Emery et al., 2000; Tang et al., 2010). We analyzed phase shifts only during the days when the flies still clearly showed rhythmic activity as a population. We were sometimes able to measure phase shifts over $5 \mathrm{~d}$, but more frequently phase shifts over $1-3 \mathrm{~d}$ were measured. Phase shifts showed no obvious transients, even during the first day, and were stable when measured over several days (Fig. 1-1 A available at https://doi.org/10.1523/JNEUROSCI.2259-17.2018. f1-1). This was the case for almost all genotypes used in this study, except for the two genotypes discussed below. We therefore feel confident that although for some genotypes we frequently had shorter measurements, we can compare these genotypes to those with robust rhythms measured systematically over $5 \mathrm{~d}$.

The photic phase response curves (PRCs) of wild-type flies show phase delays that are maximum at approximately ZT15 to ZT17, and phase advances that are maximum at approximately ZT21 (Levine et al., 1994; Suri et al., 1998). Surprisingly, $P d f^{0}$ flies could similarly very efficiently shift the phase of their behavior: both robust phase advances and phase delays could be observed (Fig. $1 A ; N \geq 4$ for ZT15, ZT19, and ZT21). This result fits with recent observations made with live calcium imaging (Liang et al., 2017). Since the M-oscillators express an additional neuropeptide, short neuropeptide-F, and are also glycinergic (HelfrichFörster et al., 2007; Frenkel et al., 2017; Liang et al., 2017), we decided to simply ablate the PDF-positive LNvs by expressing the proapoptotic gene hid (Renn et al., 1999). As expected, the morning peak of activity was lost in Pdf-GAL4/UAS-hid flies (Fig. 1B). These flies showed greater arrhythmicity than $P d f^{0}$ flies (Table 1). As a result, phase shifts could only be reliably quantified over 1-3 d. LNv-ablated flies shifted the phase of locomotor behavior by $\sim 3.5$ and $3 \mathrm{~h}$ in response to delaying and advancing light pulses, respectively, demonstrating that light-mediated behavioral phase resetting can occur in the absence of $\mathrm{M}$-oscillators (Fig. $1 C$ and Fig. $1-1 B$ available at https://doi.org/10.1523/JNEUROSCI.2259-17. 2018.f1-1; $N=3 ; t_{(4)}=0.1987, p=0.8522$, Student's $t$ test). This 
A

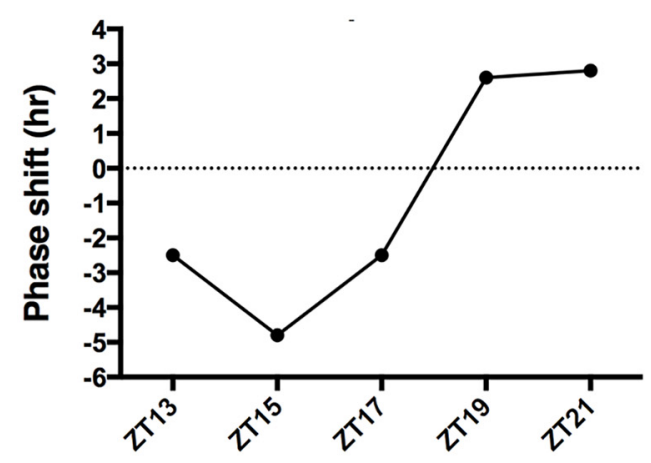

Pdfo

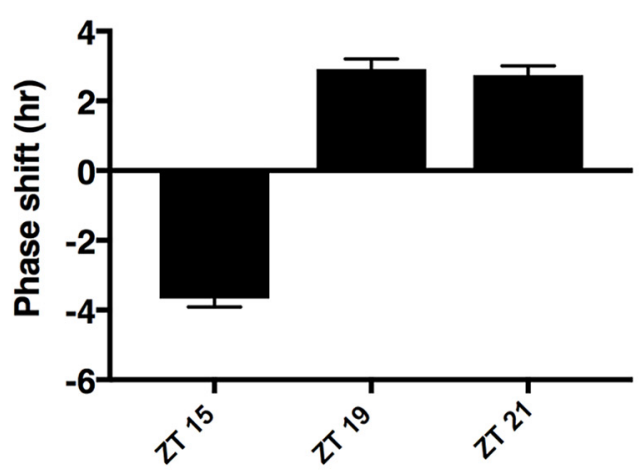

B

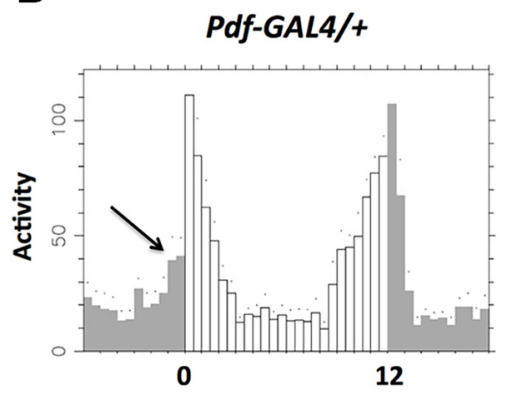

Pdf-GAL4/UAS-hid

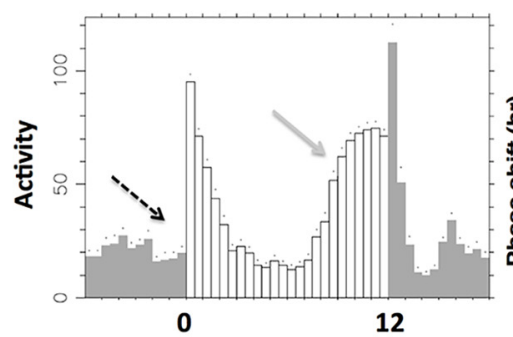

C
ZT 15

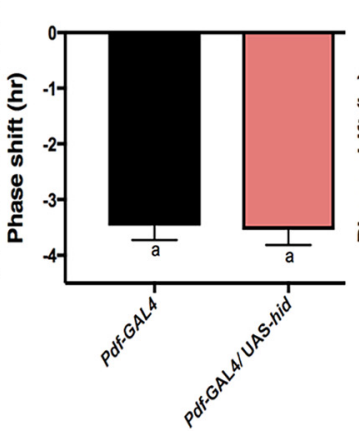

ZT 21

Figure 1. Light can reset the phase of locomotor activity in the absence of PDF-positive M-oscillators or their neuropeptide PDF. $\boldsymbol{A}, P d f^{0}$ flies can undergo phase delays as well as phase advances in response to $5 \mathrm{~min}$ light pulses (1500 lux). Since rhytmhic $P d f^{0}$ flies have a short behavior period (Table 1), their PRC could be slightly advanced compared with wild-type flies. We thus first ran a single 5 point time course (left). We then focused on the time points with maximal phase delays (ZT15) and advances [ZT19 and ZT21; right, $N$ (independent experiments) $\geq 4]$. Phase changes are plotted on the $y$-axis; phase delays and advances are represented as negative and positive values, respectively. The $x$-axis represents the ZT of the light pulse (see Fig. 1-1 A available at https://doi.org/10.1523/JNEUROSCI.2259-17.2018.f1-1). B, Ablation of M-oscillators abrogates the morning anticipation of lights-on and advances the evening peak of activity under a $12 \mathrm{~h} / 12 \mathrm{~h} \mathrm{LD}$ cycle. Activity is plotted as a function of ZT. Gray bars in the histogram represent activity levels in the night, and the white bars represent activity during the day. For Pdf-GAL4 control flies, the solid arrow shows the morning anticipatory behavior. For Pdf-GAL4/UAS-hid flies, the dashed arrow indicates the lack of morning anticipation, and the gray arrow indicates the advanced evening peak of activity. $C$, Flies with ablated M-oscillators can respond to brief light pulses. Phase delay in response to a light pulse at ZT15 is shown on the left, and phase advance in response to a light pulse at ZT21 is on the right. Both the phase delay and advance responses in Pdf-GAL4/UAS-hid flies (pink bars) were similar to the Pdf-GAL4 control flies (black bars; Fig. 1-1 B available at https://doi.org/10.1523/JNEUROSCI.2259-17.2018.f1-1). Data are plotted as the mean \pm SEM. Bars with the same letters do not significantly differ ( $p>0.05$ level, Student's $t$ test).

result, combined with our previous work (Lamba et al., 2014), also implies that the E-oscillators can shift the phase of circadian behavior without the M-oscillators.

\section{M-oscillators use PDF as a signal to modulate photic behavioral phase shifts}

The observation that the M-oscillators are dispensable for photic phase shifts seems at odds with our previous work, which had shown that CRY photoreception in the M-oscillators is required for photic behavioral phase shifts (Lamba et al., 2014). In this study, we had found that the downregulation of jet only in the PDF-positive cells compromised the ability of M-oscillators to respond to light and to trigger TIM degradation, but TIM degradation in the E-oscillators was unaffected. As a result of this manipulation, photic behavioral phase shifts were significantly weakened. To explain both these previous observations and our current observations, we hypothesized that M-oscillators lacking CRY photoreception, by not degrading TIM and thus not shifting the phase of their local oscillators, do not reset the rhythmic secretion of PDF. Thus, they compromise the resynchronization of the E-oscillators and the rest of the circadian neural network. If this were correct, flies with disrupted jet expression in the M-oscillators should phase shift normally, if they lack PDF.

As previously reported (Lamba et al., 2014), the downregulation of jet with Pdf-GAL4 compromised both phase delays and phase advances (Fig. 2A and Fig. 2-1 $A$ available at https://doi.org/ 10.1523/JNEUROSCI.2259-17.2018.f2-1). Importantly, the absence of PDF or PDFR in flies with jet knocked-down in M-oscillators completely restored normal shifts (Fig. $2 A, B$ and Fig. 2-1 $A, B$ available at https://doi.org/10.1523/JNEUROSCI. 2259-17.2018.f2-1; for Fig. 2A: ZT15, $N=4, F_{(3,12)}=22.04, p<$ 0.0001 ; ZT19, $N=6, F_{(3,20)}=10.96, p=0.0002 ; \mathrm{ZT} 21, N=5$, $F_{(4,20)}=6.174, p=0.002$, ANOVA; for Fig. $2 B: Z T 15, N \geq 3, t_{(6)}$ $=0.665, p=0.53$; ZT19, $N \geq 3, t_{(6)}=0.233, p=0.824$; ZT21, $N \geq 3, t_{(6)}=0.072, p=0.945$, Student's $t$ test). These results suggest that the circadian-blind $\mathrm{M}$-oscillators indeed inhibit photic behavioral resetting via untimely secretion of PDF.

We noticed that, at ZT19 and 21, Pdf-GAL4/UAS-jet RNAi flies usually showed a transient phase shift during the first day or two after the light pulse, which then tended to disappear (Fig. 2-1 $A$ available at https://doi.org/10.1523/JNEUROSCI.225917.2018.f2-1). This does not affect our conclusions. Indeed, 
A

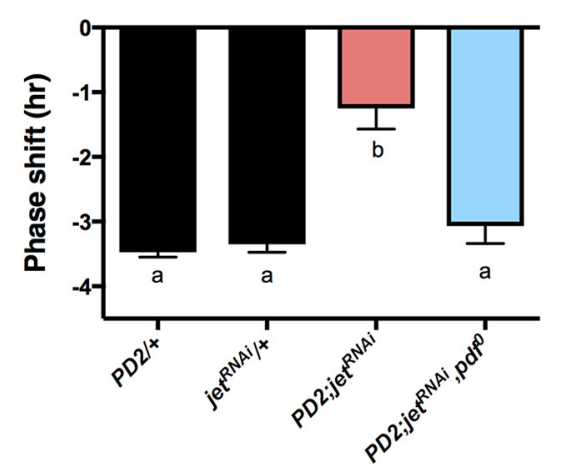

B

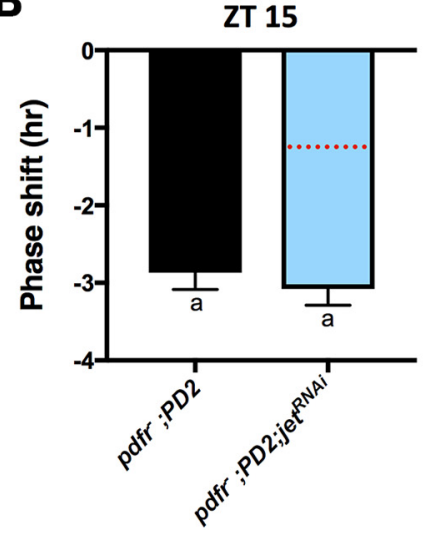

ZT 19

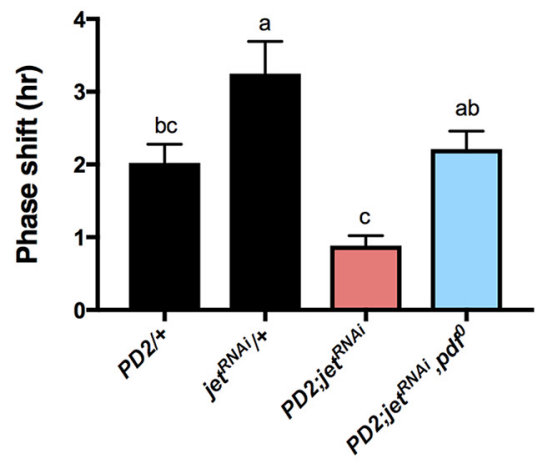

ZT 19

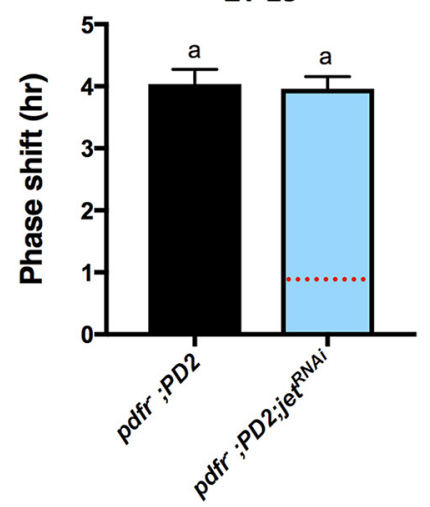

ZT 21

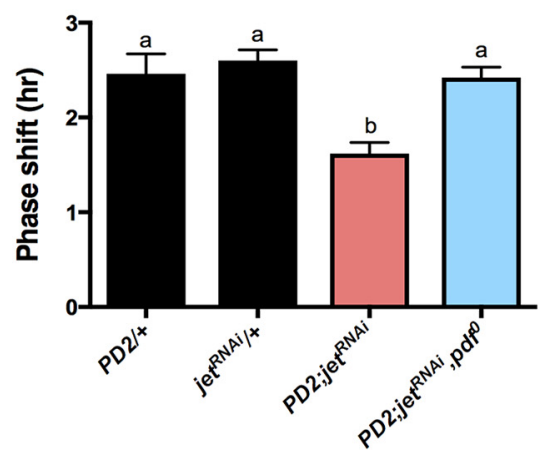

ZT 21

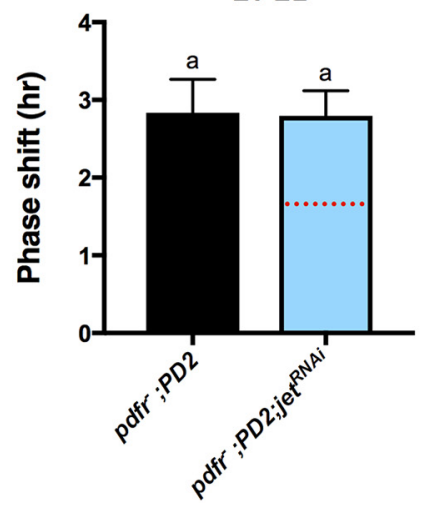

Figure 2. M-oscillators use PDF to modulate light-mediated phase responses. A, Knocking down jet in the M-oscillators (pink bar) reduces the phase delay and advance responses compared with the Pdf-GAL4 and jet RNAi control lines (black bars). Loss of PDF restores normal phase shifts in flies with jet downregulation in M-oscillators (blue bar). $N=4$ for phase responses at ZT15; $N=6$ for ZT19 and ZT21. Data represent the mean \pm SEM. Different letters above the bars indicate significant differences revealed by one-way ANOVA coupled to post hoc Tukey's test for multiple comparisons $(p<0.05$ ). PD2, Pdf-GAL4 UAS-dcr2 (Fig. 2-1 A available at https://doi.org/10.1523/JNEUROSCI.2259-17.2018.f2-1). B, Loss of PDF receptor restores normal behavioral phase shifts in flies with jet donwregulation in M-oscillators (blue bar), as they were similar to the control $p d f{ }^{-} ; P D 2$ flies (black bar). The dotted lines in the blue bars are a reminder of the average phase shift observed for PD2; jetRNAi (see $A$ ). $N \geq 3$. Data represent the mean \pm SEM. The same letters indicate no significant difference. Statistical significance was tested as described above (Fig. 2-1B available at https://doi.org/10.1523/JNEUROS(I.2259-17.2018.f2-1).

phase shifts are stable in the experimental flies lacking PDF (as assayed by quantification, when possible, or by visual inspection of the traces). If anything, differences are stronger than shown in Figure $2 A$ once a stable phase is reached.

Light can reset circadian behavior without the E-oscillators Recent studies on behavioral entrainment to LD cycles and the response of the circadian neural network in a dish to light exposure suggest a hierarchy among the circadian neurons, with the E-oscillators leading the entrainment of the network (Roberts et al., 2015; Yoshii et al., 2015). To test the significance of the E-oscillators during photoresponses, we ablated the E-oscillators by expressing UAS-hid using the cry-GAL4(13) driver combined with the $p d f$-GAL80 repressor (Stoleru et al., 2004) and administered light pulses both at ZT15 and ZT21. The efficiency of ablation of the E-oscillators was verified by the suppression of the evening peak of activity in a $12 \mathrm{~h} / 12 \mathrm{~h} \mathrm{LD}$ cycle, while protection of the $\mathrm{M}$ cells was shown by the persistence of a morning peak in activity (Fig. 3A). We found that flies with ablated E-oscillators showed high arrhythmicity (Table 1) consistent with the important role played by these cells in the control of circadian behavior (Guo et al., 2014). Surprisingly, ablation of the E-oscillators did not compromise the circadian ability of flies to sense light as they underwent a phase shift of $\sim 3 \mathrm{~h}$ both in the delay zone as well as in the advance zone (Fig. 3B and Fig. 3-1 available at https://doi.org/
10.1523/JNEUROSCI.2259-17.2018.f3-1; for ZT15, $N=4, F_{(2,8)}=$ $0.1877, p=0.8324$; for ZT21, $N=4, F_{(2,8)}=0.6536, p=0.5459$, ANOVA). We noticed, however, a transient during the first day after a pulse at ZT21 in the E-oscillator-ablated flies: the phase shift was much less pronounced on day 1 compared with the other days (Fig. 3-1, bottom available at https://doi.org/10.1523/JNEUROSCI.225917.2018.f3-1). This is probably because activity is concentrated in the morning in these flies, and the circadian network has not yet fully resynchronized. This does not impact our conclusion that the E-oscillators are not required for phase shift, since this transient slightly decreased the average phase shifts shown in Figure $3 B$.

In summary, neither the M-oscillators nor the E-oscillators are required for photic behavioral phase shifts, but, if present, both neuronal groups need to detect light for phase shifts to occur properly (see also Lamba et al., 2014). This reinforces the idea that the circadian neural network modulates cell-autonomous circadian photoresponses.

\section{Dorsal neurons can impact photic behavioral phase advances}

Since the cry-GAL4(13) driver is also expressed in some of the dorsal neurons, including the DN1as, a subset of DN1ps, and a few DN3s (Shafer et al., 2006), we decided to use a more specific GAL4 driver, DvPdf-GAL4 (combined with Pdf-GAL80), to manipulate the E-oscillators. $D v P d f$-GAL4; Pdf-GAL80 is specifically expressed in 3-4 LNds and the single fifth sLNv per brain hemi- 
A
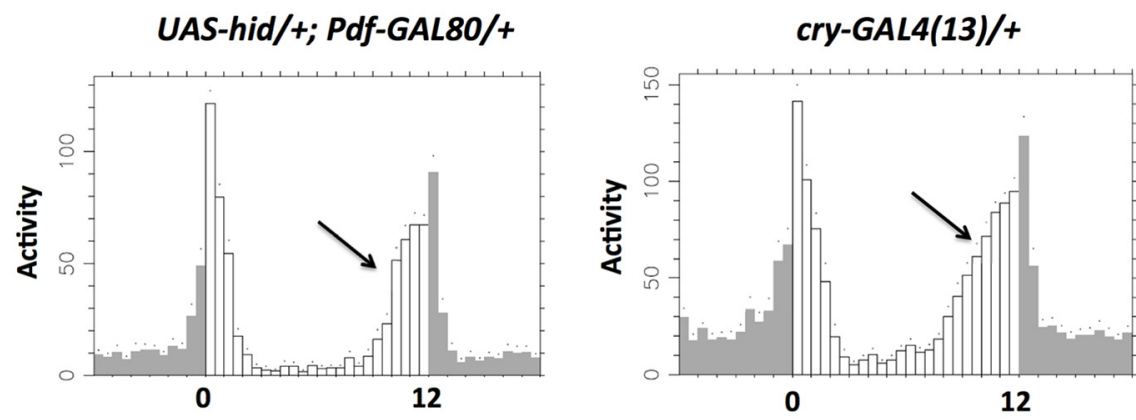

UAS-hid; Pdf-GAL80/cry-GAL4(13)

B

ZT 15

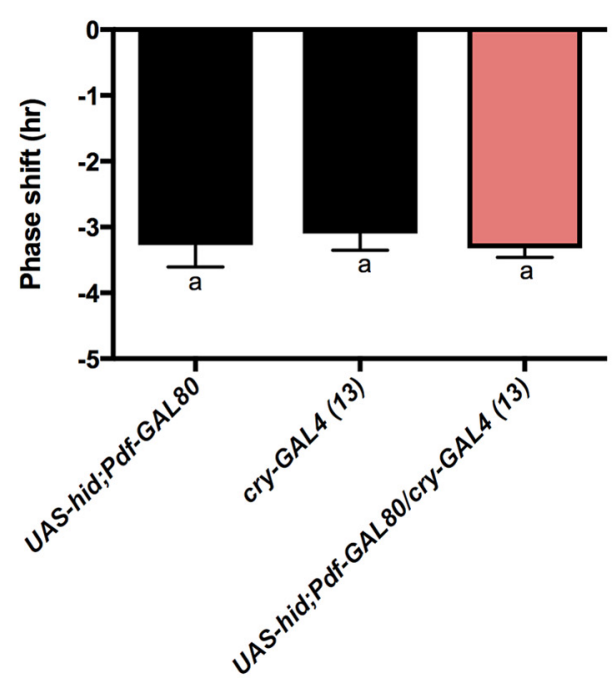

ZT 21

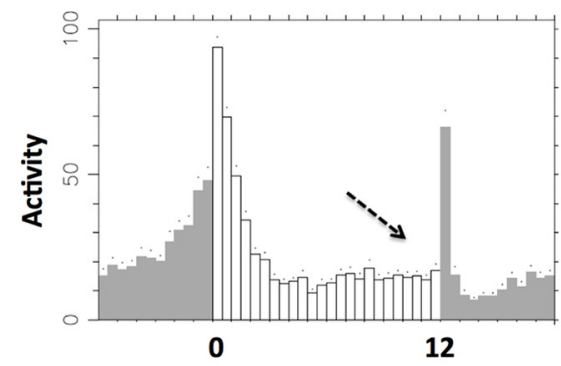

Figure 3. Light can reset the phase of circadian behavior in the absence of E-oscillators. $\boldsymbol{A}$, Ablation of E-oscillators (as well as a subset of dorsal neurons) abrogates the evening peak of activity under a $12 \mathrm{~h} / 12 \mathrm{~h} \mathrm{LD}$ cycle. The first two graphs (left and center) show the LD activity profiles of the control flies. Solid arrows indicate the evening anticipatory behavior. The dashed arrow in the last graph on the right shows the disruption of evening activity peak upon the ablation of E-cells. $\boldsymbol{B}$, Ablation of E-oscillators (pink bar) had no effect on phase delay or advance responses. Black bars are the UAS and GAL4 control flies (Fig. 3-1 available at https://doi.org/10.1523/JNEUROSCI.2259-17.2018.f3-1). N = 4. Error bars represent SEM. Bars with the same letter do not differ significantly $(p>0.05)$ by one-way ANOVA and post hoc Tukey's test.

sphere (Bahn et al., 2009; Guo et al., 2014; Lamba et al., 2014). Unfortunately, we were not able to monitor flies with Eoscillators ablated with DvPdf-GAL4; Pdf-GAL80 because these flies died 2-3 d after eclosion. Hence, we electrically silenced E-oscillators only during behavioral monitoring (LD and DD), but not during development, by conditionally expressing the inward-rectifier potassium channel KIR using tubulin (tub)$G A L 80^{t s}$ to prevent the neurons from firing action potentials at a temperature of $29^{\circ} \mathrm{C}$ (Guo et al., 2014). As previously noted (Guo et al., 2014), tub-Gal80 ${ }^{t s}$; DvPdf-GAL4/UAS-Kir; Pdf-GAL80 flies as well as $t u b-G a l 80^{t s}$; UAS-Kir control flies exhibited a long period of $\sim 26 \mathrm{~h}$ in DD (Table 1 ). This long period, and the need to expose the flies to $29^{\circ} \mathrm{C}$, delays the evening peak of activity into the night (Majercak et al., 1999). Thus, we confirmed the expected absence of the E-peak of activity under a long photoperiod $(16 \mathrm{~h} / 8 \mathrm{~h}) \mathrm{LD}$ cycle in addition to the regular $12 \mathrm{~h} / 12 \mathrm{~h} \mathrm{LD}$ cycle to be able to clearly visualize the evening peak of activity. The evening peak was observed in the control flies but not in the E-oscillator-silenced flies (Fig. 4A). To determine the effect of this silencing on phase shifts, we administered light pulses not just at ZT15 and ZT21, but also at ZT17 and ZT23 to ascertain that the maximum phase delay and advance are not missed due to the slow pace of the circadian pacemaker.
Flies with electrically silenced E-oscillators robustly shifted the phase of their circadian behavioral rhythms in response to delaying (ZT15, ZT17) light pulses. However, a marginal but statistically significant $0.5 \mathrm{~h}$ reduction in phase shift was noted at ZT17, a time at which the phase shift was maximal in both the experimental flies and the control flies with the same period length (Fig. 4B and Fig. 4-1 $A$ available at https://doi.org/10.1523/ JNEUROSCI.2259-17.2018.f4-1; at ZT15, shift amplitude was similar, but not maximal, in the two genotypes with a $26 \mathrm{~h}$ period length; for ZT15, $N=5, F_{(2,12)}=8.963, p=0.004$; For ZT17, $N=$ $5, F_{(2,12)}=5.497, p=0.02$, ANOVA). Unexpectedly, flies with electrically silenced E-oscillators showed few or no phase shifts in response to advancing light pulses at ZT21 and ZT23 (Fig. 4C and Fig. 4-1 $A$ available at https://doi.org/10.1523/JNEUROSCI.225917.2018.f4-1; for ZT21, $N=5, F_{(2,12)}=26.1, p<0.0001$; for ZT23, $N=4, F_{(2,9)}=15.54, p=0.001$, ANOVA). To ensure that this phenotype was indeed caused by conditional electrical silencing of the E-oscillators, and not by any other cause such as a subtle difference in genetic background, we pulsed the same genotypes at ZT23, but at the restrictive temperature of $20^{\circ} \mathrm{C}$ (Fig. 4D). As expected, the evening peak was now visible in the experimental flies since KIR was not expressed in the E-oscillators (Fig. 4A). Importantly, phase advances were also 
A

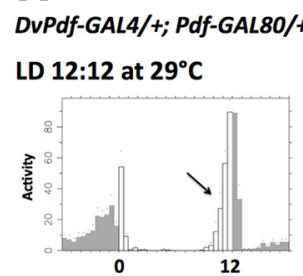

tub-GAL80ts/+; UAS-Kir/t
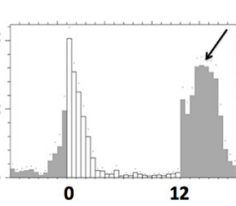

tub-GAL80ts/+; DvPdf-GAL4/

UAS-Kir; Pdf-GAL80

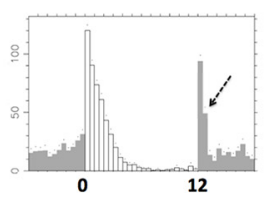

LD $16: 8$ at $29^{\circ} \mathrm{C}$
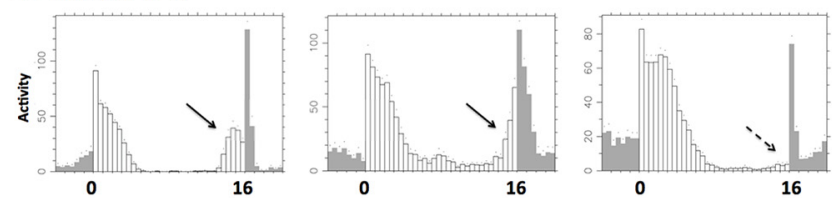

LD $16: 8$ at $20^{\circ} \mathrm{C}$
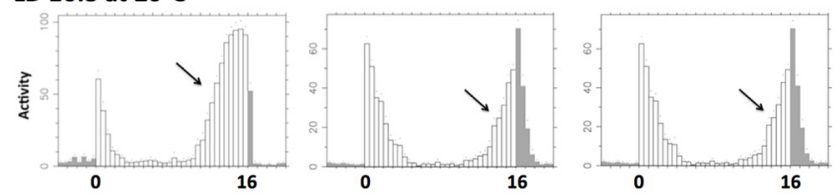

B

ZT 15

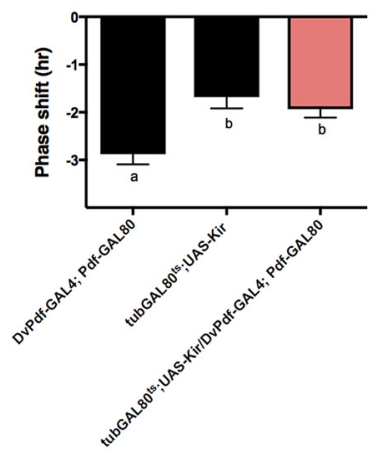

ZT 21

C

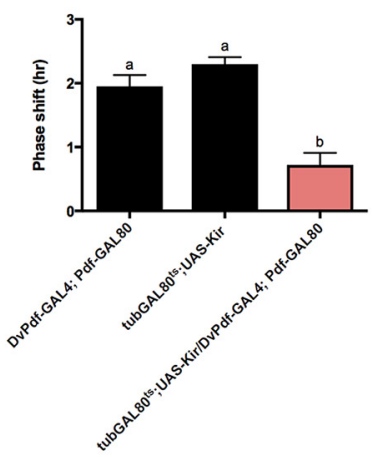

ZT 17

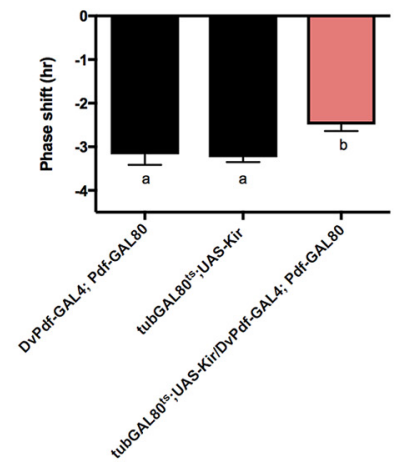

ZT 23

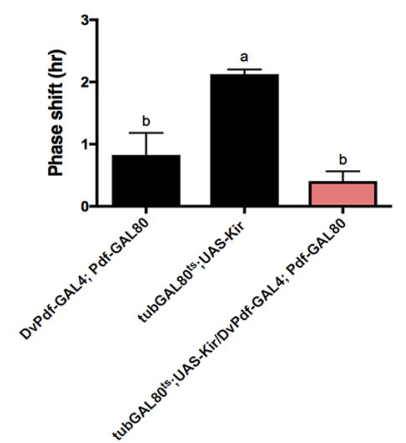

\section{ZT 23 at $20^{\circ} \mathrm{C}$}

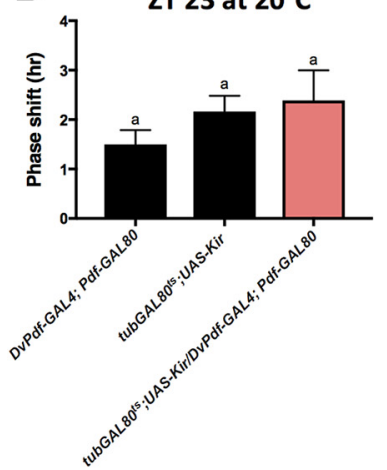

Figure 4. Phase advance responses are defective when the E-oscillators— but not the dorsal neurons—are electrically silenced. $A$, Electrical silencing of E-oscillators disrupts the evening peak of activity. The first row shows the locomotor activity under a $12 \mathrm{~h} / 12 \mathrm{~h} \mathrm{LD}$ cycle. The second row shows locomotor activity rhythms under a $16 \mathrm{~h} / 8 \mathrm{~h} \mathrm{LD}$ cycle at the permissive temperature of $29^{\circ} \mathrm{C}$ (inducing KIR expression in experimental flies), and the third row shows the activity under a $16 \mathrm{~h} / 8 \mathrm{~h} \mathrm{LD}$ cycle at the restrictive temperature of $20^{\circ} \mathrm{C}$ (no KIR expression). The evening peak of the UAS control and the experimental flies is shifted in the dark phase of the $12 \mathrm{~h} / 12 \mathrm{~h} \mathrm{LD}$ cycle because of their long circadian period of $26 \mathrm{~h}$ (Table 1), but the presence of an evening peak is clearly revealed in these two genotypes when exposed to a $16 \mathrm{~h} / 8 \mathrm{~h} \mathrm{LD}$ cycle. Note that at $20^{\circ} \mathrm{C}$, the evening peak of activity is present in control as well as in experimental flies, but it is absent in experimental flies at $29^{\circ} \mathrm{C}$. Solid arrows indicate the normal evening anticipation and dashed arrows represent abrogation of the evening peak in the electrically silenced flies. $\boldsymbol{B}$, Specific electrical inactivation of the E-oscillators does not block the response to early night light pulses. Blackbars, Control flies; pink bars, flies in which the E-oscillators are electrically silenced. The phase shift in response to a light pulse at ZT15 is shown on the left, and that for ZT17 on the right. Note that at ZT15 both the UAS control and the experimental flies have a smaller phase shift than GAL 4 control flies due to their long-period phenotype (Table 1). At ZT17, both the GAL4 and UAS controls shift normally despite the difference in period length. The E-oscillator silenced flies also phase shift in response to a ZT17 light pulse, although with a very slightly reduced amplitude, which was statistically significant from control flies as determined by one-way ANOVA followed by Tukey's test (Fig. 4-1A available at https:// doi.org/10.1523/JNEUROSCI.2259-17.2018.f4-1). $N=5$. Different letters above the bars represent significant difference between genotypes $(p<0.05)$. C, Specific electrical inactivation of the E-oscillators compromises the response to late-night light pulses. The phase response to ZT21 light pulses is shown on the left, and ZT23 on the right. Silencing the E-oscillators with the DvPdf-GAL4; Pdf-GAL80 combination (pinkbar) disrupts the phase advance response (Fig. 4-1A available at https://doi.org/10.1523/JNEUROSCI.2259-17.2018.f4-1). Note that the dorsal neurons are not affected by this manipulation. Phase shifts at ZT23 were tested to ensure that the reduction seen in experimental flies at ZT21 was not caused by the long-period phenotype of flies carrying the tub-GAL80ts; UAS-Kir combination (Table 1). ZT23 is not the time point for maximum advances for flies with a normal $\sim 24 \mathrm{~h}$ period rhythm; therefore, the GAL 4 control shows a reduced response compared with ZT21. However, the phase shifts in electrically silenced E-oscillators and the UAS control flies with a similar endogenous long period were statistically different at both ZT21 and ZT23. $N=5$. Statistical analysis was performed as described above. D. Flies carrying tub-GAL8Ots; UAS-Kir and DvPdf-GAL4; Pdf-GAL80 show a normal phase advance response at ZT23 at the restrictive temperature of $20^{\circ} \mathrm{C}$ (Fig. 4-1 B available at https://doi.org/10.1523/JNEUROSCI.2259-17.2018.f4-1). $N=3$. Statistical analysis was performed as described above.

restored (Fig. $4 D$ and $4-1 B$ available at https://doi.org/ 10.1523/JNEUROSCI.2259-17.2018.f4-1; $N=3, F_{(2,6)}=$ $1.159, p=0.375$, ANOVA).

The difference in phase shifts observed when expressing Head Involution Defective (HID) or KIR in E-oscillators could be caused by the different methods used to manipulate the E-oscillators or by the use of two drivers with slightly different expression patterns. Thus, we also electrically silenced the E-oscillators using the PdfGAL80; cry-GAL4(13) combination (Fig. 5A). However, these flies still underwent normal phase resetting in response to both phasedelaying (ZT17) as well as phase-advancing (ZT21) light pulses (Fig. $5 B$ and Fig. 5-1 available at https://doi.org/10.1523/JNEUROSCI. 2259-17.2018.f5-1; for ZT17, $N=4, F_{(2,9)}=4.69$, $p=0.64$; for ZT21, $N=4, F_{(2,9)}=13.08, p=0.002$, ANOVA). The light pulse was administered at ZT17 instead of ZT15 for phase delay responses because of the long-period length for $t u b-G a l 80^{t s}$; UASKir/Pdf-GAL80; cry-GAL4(13) and the tub-Gal80 ${ }^{\text {ts }}$; UAS-Kir control flies. For phase advance responses, the light pulse was 
A

LD 12:12
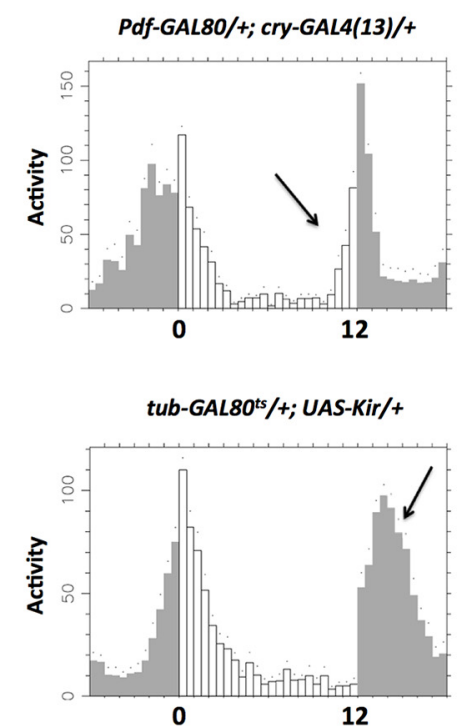

tub-GAL8Ots/t; UAS-Kir/Pdf-GAL80; cry-GAL4(13)

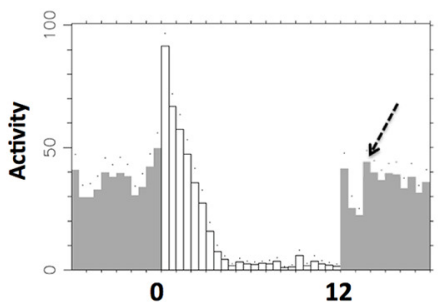

LD 16:8
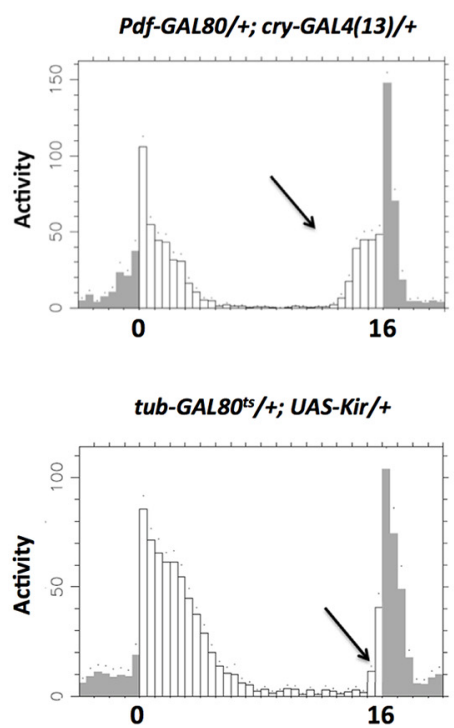

tub-GAL80ts/+; UAS-Kir/Pdf-GAL80; cry-GAL4(13)

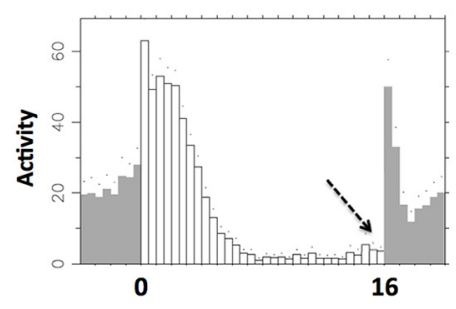

B

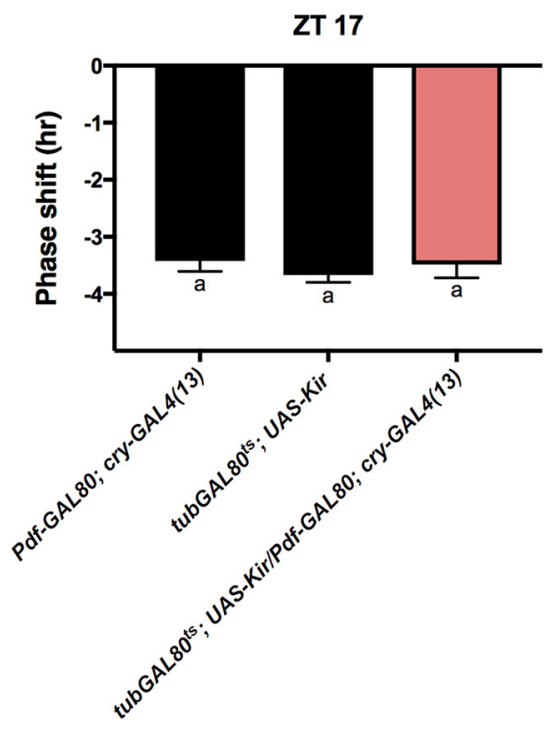

ZT 21

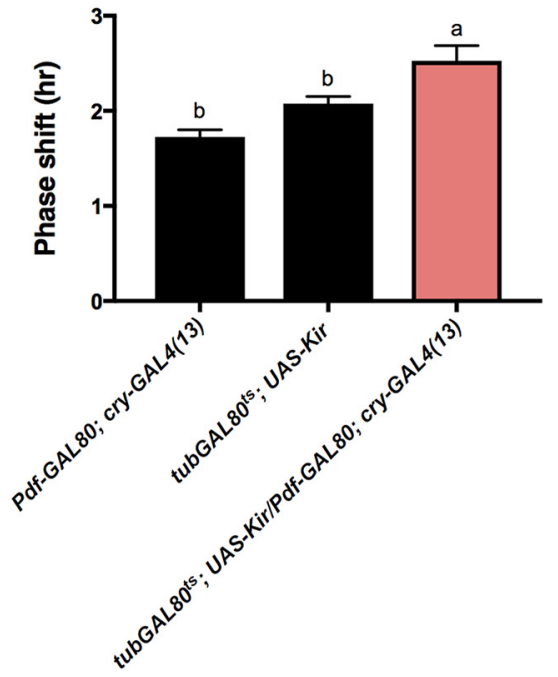

Figure 5. Light can reset the phase of circadian behavior when E-oscillators as well as a subset of dorsal neurons are electrically silenced. A, Inhibition of firing from E-oscillators and a subset of dorsal neurons using the Pdf-GAL80; cry-GAL4(13) combination prevented the evening anticipation peak. The column on the left shows the locomotor activity in the $12 \mathrm{~h} / 12 \mathrm{~h} L \mathrm{D}$ cycle and the second column on the right shows activity in $16 \mathrm{~h} / 8 \mathrm{~h} \mathrm{LD}$ cycle. Solid arrows indicate evening anticipation, and dashed arrows indicate loss of the evening peak. B, Silencing the E-oscillators with Pdf-GAL80; cry-GAL4(13) does not reduce the phase delay response to ZT17 light pulses (top) or the phase advance response to ZT21 light pulses (bottom; Fig. 5-1 available at https://doi.org/10.1523/ JNEUROSCI.2259-17.2018.f5-1). Black bars, Control flies; pink bar, electrically silenced flies. $N=4$. Error bars represent the SEM.

still administered at ZT21, since even the experimental flies showed robust phase shifts. Thus, silencing the E-oscillators as well as a subset of dorsal neurons does not impact circadian phase shifts, while sparing the dorsal neurons from silencing reduces photic behavioral phase shifts, particularly phase advances. Thus, under certain circumstances, a subset of dorsal neurons can inhibit behavioral phase shifts.

The photic phase response curve of peripheral oscillators is similar to the behavioral PRC

The behavioral PRC of Drosophila has been well characterized (Levine et al., 1994; Suri et al., 1998). It is a type I PRC with delays in the early night, advances in the late night, and a crossover point in the middle of the night (ZT18). The PRC is certainly shaped by cell-autonomous TIM degradation (Suri et al., 1998; Yang et al., 1998), but could also be influenced by neural interaction. The results presented above show that neither the M-oscillators nor the E-oscillators are required for phase delays or advances. This suggests that the properties of the PRC are essentially driven by cell-autonomous mechanisms, and neural network interaction has little impact on the shape of the PRC. Furthermore, the PRC of $P d f^{0-l}$ mutant flies (Fig. $1 A$ ) is essentially the same as that of wild-type flies (Levine et al., 1994; Suri et al., 1998). To test the hypothesis that the shape of the PRC is essentially a cellautonomous property, we turned to peripheral oscillators, which respond to light in an autonomous, brain-independent manner (Plautz et al., 1997; Emery et al., 2000; Levine et al., 2002). In flies expressing a TIM-LUC fusion gene under the control of the tim promoter, the LUC signal primarily comes from peripheral clocks of the abdomen (Fig. $6 A$; for ZT18, $N=8, t_{(14)}=4.577$, $p=0.0004$; for ZT19, $N=6, t_{(10)}=4.384, p=0.0014$, Student's $t$ test). Confirming that these peripheral clocks entrain to light autonomously, we observed both phase delays and advances of LUC rhythms in decapitated bodies in response to short light pulses (Fig. 6-1 $A$ available at https://doi.org/10.1523/JNEUROSCI. 2259-17.2018.f6-1; $N=3$ ). However, due to the poor quality of 

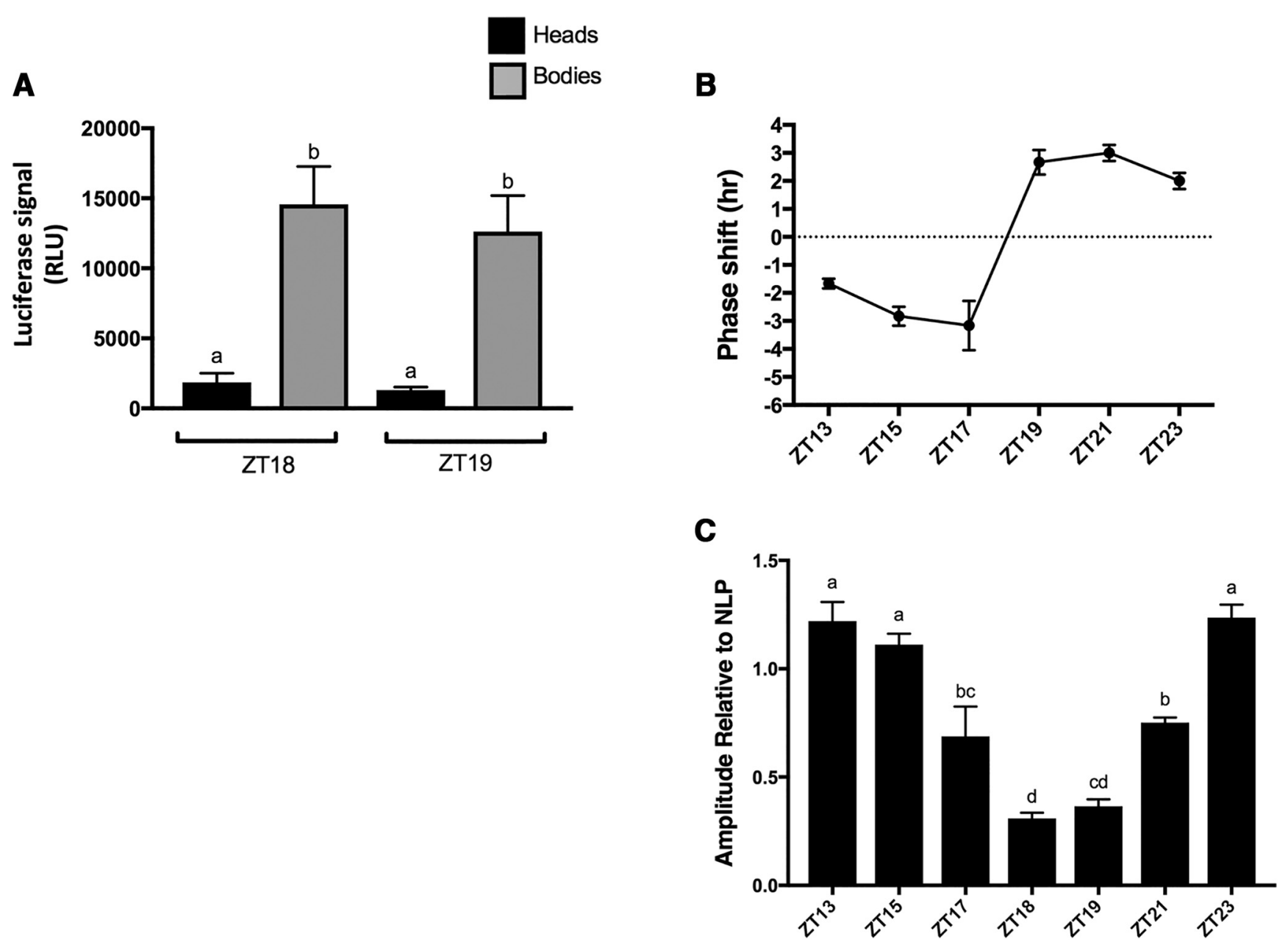

Figure 6. Photic phase responses in peripheral oscillators resemble those of circadian behavior. A, Whole fly luciferase signal generated by the ptim-TIM-LUC transgene primarily comes from peripheral tissues. Both at ZT18 and ZT19 (time points for the peak TIM-LUC bioluminescence), the majority of the TIM-LUC signal is emitted from the bodies, with only a small contribution from the heads. The difference between the TIM-LUC signal from heads and bodies is statistically significant, as determined by Student's $t$ test at both time points. $\boldsymbol{B}$, Phase response curve of luciferase rhythms in whole ptim-TIM-LUC flies. Sixteen flies were tested for each time point and in each experiment. Phase shifts in the TIM-LUC bioluminescence rhythms are plotted on the $y$-axis, and the time at which the light pulse was administered is on the $x$-axis ( $N=3$; Fig. 6-1 A-D available at https://doi.org/10.1523/JNEUROSCI.2259-17.2018.f6-1). C, Amplitude of TIM-LUC rhythms. The amplitude of the light-pulsed flies is plotted relative to non-light-pulsed (NLP) flies on the $y$-axis. $x$-axis, Different ZTs for light pulses. Note: luciferase rhythm amplitude is significantly reduced when the light pulse is administered at time points closer to the middle of the night (Fig. 6-1 D available at https://doi.org/10.1523/JNEUROSCI.2259-17.2018.f6-1). Different letters indicate significant difference as determined by ANOVA followed by post hoc Tukey's test, $p<0.05$.

these rhythms and the need to use a large number of bodies (see Materials and Methods), we measured the PRC of peripheral tissues in intact flies. Since, even with intact flies, the LUC rhythm amplitude rapidly decreased in constant conditions, we used only the first peak of luciferase activity to measure phase shifts. However, in most traces, the subsequent trough and peak also showed a similar phase shift. The photic PRC for peripheral TIM-LUC rhythms, determined at saturating light intensity (Fig. 6-1 B available at https://doi.org/10.1523/JNEUROSCI.2259-17.2018.f6-1), was strikingly similar to the behavioral PRC, showing type I PRC properties (Fig. $6 B$ and Fig. 6-1C available at https://doi. org/10.1523/JNEUROSCI.2259-17.2018.f6-1; $N=3$ ). We measured light responses at ZT18, and observed severely attenuated TIM-LUC rhythms after the light pulses, (Fig. $6 C$ and Fig. 6-1D available at https://doi.org/10.1523/JNEUROSCI.2259-17.2018. f6-1), which made measurements of phase shifts arduous. However, no clear phase shift could be observed visually. Interestingly, rhythm amplitudes were also reduced at ZT19, but they were clearly phase advanced. A small decrease in amplitude was also noted with pulses at ZT17 and ZT21 (Fig. 6C). The similarity between behavioral and peripheral PRCs strengthens the idea that properties of the Drosophila photic PRC rely largely on cellautonomous resetting mechanisms.

\section{Discussion}

The model for light-mediated resetting of the Drosophila molecular circadian pacemaker posits that circadian photoreception is cell autonomous. Indeed, tim mRNA and protein cycles combined with CRY-dependent TIM degradation provide a simple and reasonably satisfactory explanation for how circadian phase delays and advances can be achieved cell autonomously (Ashmore and Sehgal, 2003). In fact, the ectopic expression of CRY in the ovaries can cause TIM degradation upon exposure to light, which is otherwise light insensitive in these organs (Rush et al., 2006), and adding CRY to blind larval DN2s anchors the phase of their molecular oscillator to the LD cycle (Klarsfeld et al., 2004). However, we, as well as others, have recently shown that neural interactions are required for proper circadian resetting of brain clocks and circadian behavior (Shang et al., 2008; Tang et al., 2010; Lamba et al., 2014; Roberts et al., 2015; Yoshii et al., 2015). 
It has been proposed that defined groups of circadian neurons specifically promote phase advances or delays (Shang et al., 2008; Tang et al., 2010). However, our present results support the idea that behavioral phase advances and delays induced by light input are derived from the molecular properties of the circadian clock and CRY photoreception. We had previously shown that CRY photoreception is required in both the $\mathrm{M}$ - and E-oscillators, and that these neurons cooperate to reset circadian behavior (Lamba et al., 2014). However, we find here that neither ablation of the M-oscillators nor elimination of the E-oscillators (with some dorsal neurons; see below) impacts the ability of the flies to advance or delay their circadian behavior. Combined with our previous work, this indicates that the M-oscillators or the E-oscillators, on their own, can generate both phase advances or phase delays, although we cannot exclude the possibility that dorsal neurons can substitute for the loss of either group of cells. However, our finding that the PRC of peripheral oscillators, which are not part of a neuronal network and are able to entrain to light autonomously through CRY (Plautz et al., 1997; Emery et al., 2000; Levine et al., 2002), is so similar to that of the behavioral PRC strongly supports the hypothesis that the properties of CRY entrainment are essentially derived from its cell-autonomous nature, and this is also true in the brain. Of particular interest is the response of peripheral oscillators to light pulses administered at ZT18. There is no clear phase shift, which supports the idea that the peripheral PRC, similar to behavior (brain) PRC, is a type 1 PRC (a type 0 PRC would have a strong phase response to light pulses administered near the middle of the night). There is, however, another striking feature to the ZT18 response: an important loss in oscillation amplitude. This can be explained in two ways. First, a pulse at ZT18 dampens circadian oscillations in peripheral oscillators. Indeed, limit cycle theory predicts that a pulse of the appropriate magnitude at the correct time can stop circadian oscillations. This phenomenon has been observed in multiple organisms, including Drosophila pseudoobscura, Neurospora, and humans (Winfree, 1970; Jewett et al., 1991; Huang et al., 2006). Alternatively, the loss of amplitude could be caused by phase dispersion, with some oscillators delaying, and some advancing, their phase in response to a light pulse occurring so close to the crossover point of the PRC.

Although our results support the idea that the properties of the behavioral PRC emanate from the cell-autonomous nature of circadian photoreception, it is clear that, in the brain, neuronal interactions play an important role. First, nonautonomous acute TIM degradation is observed in the brain upon light exposure (Yoshii et al., 2008; Lamba et al., 2014). Second, proper interaction in the $\mathrm{M}$ - and E-oscillator network is clearly critical for lightmediated phase resetting. Indeed, combined with our previous work (Lamba et al., 2014), our present results indicate that both $\mathrm{M}$ - and E-oscillators need to be properly reset. If these oscillatory groups are not in unison, conflicting signals being sent from the $\mathrm{M}$ - and $\mathrm{E}$-oscillators prevent normal resetting of the circadian clock network. Indeed, removing PDF in flies in which JET expression is compromised in M-oscillators restores normal phase shifts (Fig. 2A). In these flies, untimely secreted PDF thus inhibits circadian behavioral photoresponses. The nature of the neurotransmitters from the E-oscillators that would similarly reduce behavioral phase shifts, when those cells are defective for JET, remains to be determined. Thus, we propose that, in a normal circadian circuit, proper synchronization of local phase resetting in $\mathrm{M}$ and $\mathrm{E}$-oscillators is critical to coordinate circadian behavioral photoresponses. The latter proposal seems to be at odds with observations made by Guo et al. (2014). Indeed, these authors found that thermogenetic activation of the M-oscillators using the temperature sensor TRPA1 (transient receptor potential cation channel A1) is sufficient to trigger phase advances and delays similar to those triggered by light pulses. These phase shifts were PDF dependent. It is entirely possible, however, that thermogenetic activation of $\mathrm{M}$-oscillators triggers a much more intense firing activity than light pulses and thus greater PDF release, which could override the absence of direct activation of the E-oscillators. Third, although circadian photoreception is neither necessary nor sufficient in the lLNvs (Lamba et al., 2014), elimination of these cells in otherwise wild-type flies compromises phase advances (Shang et al., 2008). Moreover, we uncovered evidence implicating dorsal neurons in phase advances as well. Indeed, while silencing the E-oscillators and a subset of dorsal neurons with the cry-GAL4(13) driver had no impact on phase advances or delays, silencing just the E-oscillators unexpectedly strongly reduced phase advances (a very weak effect on phase delays was also noted). Thus, it appears that a subset of dorsal neurons, which, based on the expression patterns of GAL4 drivers, might include the 2 DN1as, some DN1ps, and a few DN3s (Shafer et al., 2006), can inhibit phase shifts in the absence of active E-oscillators. Anatomically, some DN1 fibers project ventrally and are in close association with the dorsal projections of the M-oscillators (Zhang et al., 2010). Moreover, it has been shown that the larval DN1s, which form the 2 DN1as in adult flies, inhibit sLNv neuronal activity via glutamate to modulate light-avoidance behavior (Collins et al., 2012). Also, a functional clock only in the DN1ps can rescue the morning anticipation peak in per $^{0}$ flies and suppress the morning peak of activity under low ambient temperature, suggesting that the DN1ps can feed back on the M-oscillators to regulate their output (Zhang et al., 2010). Thus, there is ample evidence that the DN1s can influence activity of the M-oscillators. It is not clear under which circumstances dorsal neurons intervene in the regulation of circadian light responses in a wild-type fly at this point, but such a function might contribute to the plasticity of circadian behavior to environmental inputs.

In summary, we propose that properties of photic (CRYdependent) circadian entrainment in Drosophila emerge from the cell-autonomous nature of light perception, but circadian behavior resetting requires coordination between $\mathrm{M}$ - and $\mathrm{E}$-oscillators. Interestingly, in the case of temperature also, the M-oscillators on their own are poorly sensitive to temperature inputs and rely on dedicated circadian neurons, which are in this case CRY negative (Busza et al., 2007; Yoshii et al., 2010; Chen et al., 2015). Such reliance on nonautonomous mechanisms and a separate population of circadian neurons might allow the pacemaker M-oscillators to integrate multiple modalities, or to prioritize them, and thus generate circadian behavioral outputs that are as properly synchronized as possible with environmental cycles.

\section{References}

Ashmore LJ, Sehgal A (2003) A fly's eye view of circadian entrainment. J Biol Rhythms 18:206-216. CrossRef Medline

Bahn JH, Lee G, Park JH (2009) Comparative analysis of pdf-mediated circadian behaviors between Drosophila melanogaster and D. virilis. Genetics 181:965-975. CrossRef Medline

Busza A, Emery-Le M, Rosbash M, Emery P (2004) Roles of the two Drosophila CRYPTOCHROME structural domains in circadian photoreception. Science 304:1503-1506. CrossRef Medline

Busza A, Murad A, Emery P (2007) Interactions between circadian neurons 
control temperature synchronization of Drosophila behavior. J Neurosci 27:10722-10733. CrossRef Medline

Chen C, Buhl E, Xu M, Croset V, Rees JS, Lilley KS, Benton R, Hodge JJ, Stanewsky R (2015) Drosophila ionotropic receptor 25a mediates circadian clock resetting by temperature. Nature 527:516-520. CrossRef Medline

Collins B, Kane EA, Reeves DC, Akabas MH, Blau J (2012) Balance of activity between $\mathrm{LN}(\mathrm{v}) \mathrm{s}$ and glutamatergic dorsal clock neurons promotes robust circadian rhythms in Drosophila. Neuron 74:706-718. CrossRef Medline

Dubruille R, Murad A, Rosbash M, Emery P (2009) A constant light-genetic screen identifies KISMET as a regulator of circadian photoresponses. PLoS Genet 5:e1000787. CrossRef Medline

Emery P, So WV, Kaneko M, Hall JC, Rosbash M (1998) CRY, a Drosophila clock and light-regulated cryptochrome, is a major contributor to circadian rhythm resetting and photosensitivity. Cell 95:669-679. CrossRef Medline

Emery P, Stanewsky R, Helfrich-Förster C, Emery-Le M, Hall JC, Rosbash M (2000) Drosophila CRY is a deep brain circadian photoreceptor. Neuron 26:493-504. CrossRef Medline

Ewer J, Frisch B, Hamblen-Coyle MJ, Rosbash M, Hall JC (1992) Expression of the period clock gene within different cell types in the brain of Drosophila adults and mosaic analysis of these cells' influence on circadian behavioral rhythms. J Neurosci 12:3321-3349. CrossRef Medline

Frenkel L, Muraro NI, Beltrán González AN, Marcora MS, Bernabó G, Hermann-Luibl C, Romero JI, Helfrich-Förster C, Castaño EM, MarinoBusjle C, Calvo DJ, Ceriani MF (2017) Organization of circadian behavior relies on glycinergic transmission. Cell Rep 19:72-85. CrossRef Medline

Grima B, Chélot E, Xia R, Rouyer F (2004) Morning and evening peaks of activity rely on different clock neurons of the Drosophila brain. Nature 431:869-873. CrossRef Medline

Guo, F, Cerullo, I, Chen, X, Rosbash, M (2014) PDF neuron firing phaseshifts key circadian activity neurons in Drosophila. Elife 3:e02780. CrossRef Medline

Helfrich-Förster C, Winter C, Hofbauer A, Hall JC, Stanewsky R (2001) The circadian clock of fruit flies is blind after elimination of all known photoreceptors. Neuron 30:249-261. CrossRef Medline

Helfrich-Förster C, Yoshii T, Wülbeck C, Grieshaber E, Rieger D, Bachleitner W, Cusamano P, Rouyer F (2007) The lateral and dorsal neurons of Drosophila melanogaster: new insights about their morphology and function. Cold Spring Harb Symp Quant Biol 72:517-525. CrossRef Medline

Huang G, Wang L, Liu Y (2006) Molecular mechanism of suppression of circadian rhythms by a critical stimulus. EMBO J 25:5349-5357. CrossRef Medline

Hyun S, Lee Y, Hong ST, Bang S, Paik D, Kang J, Shin J, Lee J, Jeon K, Hwang S, Bae E, Kim J (2005) Drosophila GPCR han is a receptor for the circadian clock neuropeptide PDF. Neuron 48:267-278. CrossRef Medline

Jewett ME, Kronauer RE, Czeisler CA (1991) Light-induced suppression of endogenous circadian amplitude in humans. Nature 350:59-62. CrossRef Medline

Klarsfeld A, Malpel S, Michard-Vanhée C, Picot M, Chélot E, Rouyer F (2004) Novel features of cryptochrome-mediated photoreception in the brain circadian clock of Drosophila. J Neurosci 24:1468-1477. CrossRef Medline

Koh K, Zheng X, Sehgal A (2006) JETLAG resets the Drosophila circadian clock by promoting light-induced degradation of TIMELESS. Science 312:1809-1812. CrossRef Medline

Lamba P, Bilodeau-Wentworth D, Emery P, Zhang Y (2014) Morning and evening oscillators cooperate to reset circadian behavior in response to light input. Cell Rep 7:601-608. CrossRef Medline

Levine JD, Casey CI, Kalderon DD, Jackson FR (1994) Altered circadian pacemaker functions and cyclic AMP rhythms in the Drosophila learning mutant dunce. Neuron 13:967-974. CrossRef Medline

Levine JD, Funes P, Dowse HB, Hall JC (2002) Advanced analysis of a cryptochrome mutation's effects on the robustness and phase of molecular cycles in isolated peripheral tissues of Drosophila. BMC Neurosci 3:5. CrossRef Medline

Liang X, Holy TE, Taghert PH (2017) A series of suppressive signals within the Drosophila circadian neural circuit generates sequential daily outputs. Neuron 94:1173-1189.e4. CrossRef Medline

Lin FJ, Song W, Meyer-Bernstein E, Naidoo N, Sehgal A (2001) Photic signaling by cryptochrome in the Drosophila circadian system. Mol Cell Biol 21:7287-7294. CrossRef Medline
Lin Y, Stormo GD, Taghert PH (2004) The neuropeptide pigmentdispersing factor coordinates pacemaker interactions in the Drosophila circadian system. J Neurosci 24:7951-7957. CrossRef Medline

Majercak J, Sidote D, Hardin PE, Edery I (1999) How a circadian clock adapts to seasonal decreases in temperature and day length. Neuron 24: 219-230. CrossRef Medline

Ni JD, Baik LS, Holmes TC, Montell C (2017) A rhodopsin in the brain functions in circadian photoentrainment in Drosophila. Nature 545:340 344. CrossRef Medline

Plautz JD, Kaneko M, Hall JC, Kay SA (1997) Independent photoreceptive circadian clocks throughout Drosophila. Science 278:1632-1635. CrossRef Medline

Renn SC, Park JH, Rosbash M, Hall JC, Taghert PH (1999) A pdf neuropeptide gene mutation and ablation of PDF neurons each cause severe abnormalities of behavioral circadian rhythms in Drosophila. Cell 99:791-802. CrossRef Medline

Rieger D, Stanewsky R, Helfrich-Förster C (2003) Cryptochrome, compound eyes, Hofbauer-Buchner eyelets, and ocelli play different roles in the entrainment and masking pathway of the locomotor activity rhythm in the fruit fly Drosophila melanogaster. J Biol Rhythms 18:377-391. CrossRef Medline

Roberts L, Leise TL, Noguchi T, Galschiodt AM, Houl JH, Welsh DK, Holmes TC (2015) Light evokes rapid circadian network oscillator desynchrony followed by gradual phase retuning of synchrony. Curr Biol 25:858-867. CrossRef Medline

Rosato E, Trevisan A, Sandrelli F, Zordan M, Kyriacou CP, Costa R (1997) Conceptual translation of timeless reveals alternative initiating methionines in Drosophila. Nucleic Acids Res 25:455-458. CrossRef Medline

Rush BL, Murad A, Emery P, Giebultowicz JM (2006) Ectopic CRYPTOCHROME renders TIM light sensitive in the Drosophila ovary. J Biol Rhythms 21:272-278. CrossRef Medline

Shafer OT, Helfrich-Förster C, Renn SC, Taghert PH (2006) Reevaluation of Drosophila melanogaster's neuronal circadian pacemakers reveals new neuronal classes. J Comp Neurol 498:180-193. CrossRef Medline

Shang Y, Griffith LC, Rosbash M (2008) Light-arousal and circadian photoreception circuits intersect at the large PDF cells of the Drosophila brain. Proc Natl Acad Sci U S A 105:19587-19594. CrossRef Medline

Stanewsky R, Kaneko M, Emery P, Beretta B, Wager-Smith K, Kay SA, Rosbash M, Hall JC (1998) The cryb mutation identifies cryptochrome as a circadian photoreceptor in Drosophila. Cell 95:681-692. CrossRef Medline

Stoleru D, Peng Y, Agosto J, Rosbash M (2004) Coupled oscillators control morning and evening locomotor behaviour of Drosophila. Nature 431: 862-868. CrossRef Medline

Stoleru D, Peng Y, Nawathean P, Rosbash M (2005) A resetting signal between Drosophila pacemakers synchronizes morning and evening activity. Nature 438:238-242. CrossRef Medline

Suri V, Qian Z, Hall JC, Rosbash M (1998) Evidence that the TIM light response is relevant to light-induced phase shifts in Drosophila melanogaster. Neuron 21:225-234. CrossRef Medline

Tang CH, Hinteregger E, Shang Y, Rosbash M (2010) Light-mediated TIM degradation within Drosophila pacemaker neurons (s-LNvs) is neither necessary nor sufficient for delay zone phase shifts. Neuron 66:378 -385. CrossRef Medline

Winfree AT (1970) Integrated view of resetting a circadian clock. J Theor Biol 28:327-374. CrossRef Medline

Yang Z, Emerson M, Su HS, Sehgal A (1998) Response of the timeless protein to light correlates with behavioral entrainment and suggests a nonvisual pathway for circadian photoreception. Neuron 21:215-223. CrossRef Medline

Yoshii T, Todo T, Wülbeck C, Stanewsky R, Helfrich-Förster C (2008) Cryptochrome is present in the compound eyes and a subset of Drosophila's clock neurons. J Comp Neurol 508:952-966. CrossRef Medline

Yoshii T, Hermann C, Helfrich-Förster C (2010) Cryptochrome-positive and -negative clock neurons in Drosophila entrain differentially to light and temperature. J Biol Rhythms 25:387-398. CrossRef Medline

Yoshii T, Hermann-Luibl C, Kistenpfennig C, Schmid B, Tomioka K, Helfrich-Förster C (2015) Cryptochrome-dependent and -independent circadian entrainment circuits in Drosophila. J Neurosci 35:6131-6141. CrossRef Medline

Zhang Y, Liu Y, Bilodeau-Wentworth D, Hardin PE, Emery P (2010) Light and temperature control the contribution of specific DN1 neurons to Drosophila circadian behavior. Curr Biol 20:600-605. CrossRef Medline 